Ann. Biol. anim. Bioch. Biophys., 1979, 19 (2 B), 483-485.

\title{
A. Recherches anatomopathologiques chez le rat ingérant différentes doses d'huile d'arachide ou d'huile de colza à faible teneur en acide érucique (Huile de colza Primor).
}

\section{Croissance des animaux et poids des organes.}

par G. ROCQUELIN

Station de Recherches sur la Qualité des Aliments de l'Homme, I.N.R.A.,

7, rue Sully, 21034 Dijon Cedex.

Summary. A. Anatomo-pathological research on rats ingesting different doses of peanut oil or of low-erucic acid rapeseed oil (Primor rapeseed oil). 2. Animal growth and organ weight.

After 6 months of diet, the type of oil had no influence on the weight of the body and the myocardium of rats. Relative liver and kidney weights were slightly higher in the « Primor rapeseed oil » lot than in the «peanut oil » lot. After 1 year of diet, the weight of the former lot was 7 p. 100 lower than that of the latter lot. On the contrary, relative heart and liver weights of the «Primor » group were higher than those of the « peanut oil » group.

\section{Matériel et méthodes.}

Les conditions expérimentales ont élé décrites au chapitre précédent.

Les calculs statistiques ont été effectués par la méthode de l'analyse de variance à 2 voies en prenant comme critère de classification, d'une part, la nature de l'huile (arachide, colza Primor), d'autre part, la dose d'huile dans le régime (5, 10, 15 p. 100). Par cette méthode, on peut déterminer l'interaction éventuelle entre les effets dus à chacun de ces 2 critères.

\section{Résultats.}

1. Consommation de nourriture (tabl. 1).

\section{TABLEAU 1}

Consommation de nourrifure (calories ingérées/rat/jour, moyennes \pm écart type commun, $n=10$ )

Nature de l'huile

Dose (p. 100)

\begin{tabular}{ccc}
\hline 5 & 10 & 15 \\
$71,0 \pm 1,2$ & $74,1 \pm 1,2$ & $76,4 \pm 1,2$ \\
$75,3 \pm 1,2$ & $73,5 \pm 1,2$ & $75,7 \pm 1,2$
\end{tabular}

\begin{tabular}{|c|c|c|}
\hline $\begin{array}{l}\text { Arachide } \ldots \ldots \ldots \ldots \ldots \ldots \ldots \ldots \ldots \\
\text { Colza Primor } \ldots \ldots \ldots \ldots \ldots \ldots \ldots\end{array}$ & $\begin{array}{l}71,0 \pm 1,2 \\
75,3 \pm 1,2\end{array}$ & $\begin{array}{l}74,1 \pm 1,2 \\
73,5 \pm 1,2\end{array}$ \\
\hline
\end{tabular}


Pendant la période où les mesures de consommation de nourriture ont été effectuées, nous n'avons pas constaté d'influence de la nature de l'huile sur le niveau des ingérés. Nous avons seulement remarqué que les animaux soumis aux 2 régimes renfermant 5 p. 100 d'huile ingèrent plus de matière sèche que ceux des autres lots $(P<0,01)$. Si on exprime les résultats en calories ingérées, il n'y a plus de différence entre les lots (tabl. 1).

\section{Croissance corporelle.}

Après 6 mois, il n'y a pas d'influence de la nature de l'huile sur le poids corporel des animaux. Seuls les rats du lot « 5 p. 100 arachide » ont un poids corporel légèrement inférieur $(P<0,01)$ a celui des animaux des lots renfermant 10 ou 15 p. 100 d'huile dans le régime (tabl. 2).

\section{TABLEAU 2}

Poids corporel (en g) des animaux après 6 mois ef un an de mise au régime (moyennes \pm écart type commun, $n=20$ )

\begin{tabular}{|c|c|c|c|}
\hline \multirow{2}{*}{ Nature de l'huile } & \multicolumn{3}{|c|}{ Dose (p. 100) } \\
\hline & 5 & 10 & 15 \\
\hline \multicolumn{4}{|l|}{ Arachide } \\
\hline 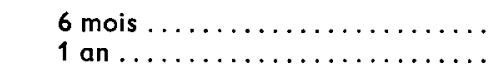 & $\begin{array}{l}536 \pm 10 \\
653 \pm 14\end{array}$ & $588 \pm 10$ & $\begin{array}{c}575 \pm 10 \\
-\end{array}$ \\
\hline \multicolumn{4}{|l|}{ Colza Primor } \\
\hline $\begin{array}{l}6 \text { mois } \ldots \ldots \ldots \ldots \ldots \ldots \ldots \ldots \ldots \ldots \ldots \ldots \ldots \\
1 \text { an } \ldots \ldots \ldots \ldots \ldots \ldots \ldots\end{array}$ & $\begin{array}{l}560 \pm 10 \\
610 \pm 14\end{array}$ & $561 \pm 10$ & $575 \pm 10$ \\
\hline
\end{tabular}

Après un an de mise au régime, on constate que les rats ingérant le régime à 5 p. 100 d'huile de colza Primor ont un poids corporel inférieur d'environ 7 p. 100 $(P<0,01)$ à celui des animaux « 5 p. 100 arachide » (tabl. 2).

3. Poids des organes après 6 mois de mise au régime.

La nature et la teneur de l'huile dans le régime sont sans effet sur le poids du myocarde.

TABLEAU 3

Poids relatif du foie après 6 mois de mise ou régime (moyennes \pm écart type commun, $n=12$ )

Nature de l'huile

Dose (p. 100)

$5 \quad 10 \quad 15$

Arachide.

$3,08 \pm 0,06$

Colza Primor

$3,29 \pm 0,06$

$2,86 \pm 0,06$

$3,19 \pm 0,06$

$2,59 \pm 0,06$

$2,97 \pm 0,06$ 
Le poids relatif du foie (poids de l'organe $\times 100 /$ poids de l'animal) est légèrement plus élevé $(P<0,01)$ chez les animaux des lots « colza Primor " que chez ceux des lots " arachide ", quelle que soit la dose d'huile dans le régime (tabl. 3). On trouve également un effet significatif $(P<0,01)$ de la dose d'huile dans le régime ; lorsque celle-ci augmente, le poids relatif du foie diminue.

Le poids relatif des reins est également légèrement, mais significativement, augmenté chez les animaux ingérant de l'huile de colza Primor $(P<0,01)$.

4. Poids des organes après un an de mise au régime.

Les poids relatifs du couv et du foie des rats soumis aux régimes contenant de I'huile de colza Primor sont significativement plus élevés $(P<0,05$ et $P<0,01)$ que ceux des rats des lots « huile arachide " (tabl. 4).

\section{TABLEAU 4}

Poids relatifs du foie ef du cœur après un on d'alimentation avec un régime contenant 5 p. 100 d'huile (moyennes \pm écart type commun, $\mathrm{n}=11$ )

\begin{tabular}{|c|c|c|}
\hline \multirow{2}{*}{ Nature de l'huile } & \multicolumn{2}{|c|}{ Organe } \\
\hline & Foie & Cœur \\
\hline $\begin{array}{l}\text { Arachide .... } \\
\text { Colza Primor }\end{array}$ & $\begin{array}{l}2,85 \pm 0,09 \\
3,34 \pm 0,09\end{array}$ & $\begin{array}{l}0,23 \pm 0,008 \\
0,26 \pm 0,008\end{array}$ \\
\hline
\end{tabular}

L'augmentation du poids du foie et des reins chez les rats ingérant de l'huile de colza très pauvre en acide érucique (huile de colza Primor) avait déjà été constatée lors de précédents travaux effectués dans notre laboratoire (Rocquelin et Cluzan, 1968 ; Nitou, 1976).

Effets physiologiques comparés des huiles de colza et d'arachide. ATP-INRA, 1974-1976.

\section{Références}

ROCQUELIN G., CLUZAN R., 1968. L'huile de colza riche en acide érucique ef l'huile de colza sans acide érucique : valeur nutritionnelle et effets physiologiques chez le rat. Ann. Biol. anim. Bioch. Biophys., 8, 395-406.

NITOU J. G., 1976. Effets propres ef inferactions entre les effets de l'huile de colza (riche ou paurre en acide érucique) et de l'éthanol sur les lipides et sur l'anatomie de quelques fissus et organes du rat. Thèse de $3^{\text {e }}$ cycle, Univ. de Dijon, 108 pp. 